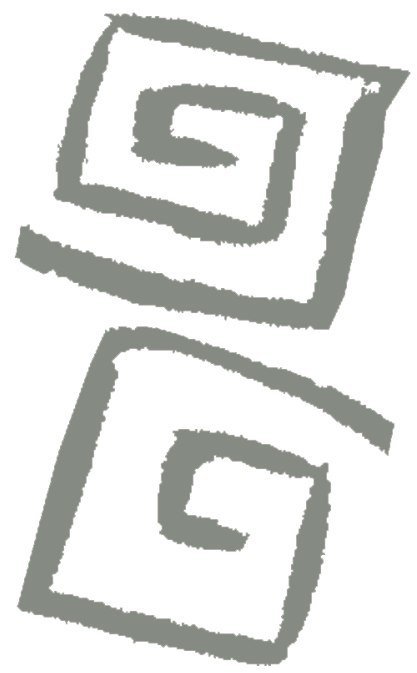

\title{
Comunicación y salud mental: características relacionadas con la empatía en médicas y médicos del primer nivel de atención en Chapecó, Santa Catarina, Brasil
}

\author{
Communication and Mental Health: Characteristics \\ related to empathy in primary care physicians in \\ Chapecó, Santa Catarina, Brazil
}

Jane Kelly Oliveira Friestino', Deoclécio Luchini Junior², Laura Lange Biesek², Grasiela Marcon ${ }^{4}$, Graciela Soares Fonsêca ${ }^{5}$

'Doctora en Salud Colectiva, área Epidemiología. Profesora Adjunta, Universidade Federal da Fronteira Sul; Chapecó, Brasil. $\triangle$ iD

${ }^{2}$ Académico, Universidade Federal da Fronteira Sul, Chapecó, Brasil. $\bowtie$ iD

${ }^{3}$ Académica, Universidade Federal da Fronteira Sul, Chapecó, Brasil. $\bowtie$ (iD)

${ }^{4}$ Magíster en Ciencias Médicas, área Psiquiatría; Profesora Asistente Universidade Federal da Fronteira Sul, Chapecó, Brasil. $\square$ (iD)

${ }^{5}$ Doctora en Ciencias Odontológicas. Profesora Adjunta, Universidade Federal da Fronteira Sul; Chapecó, Brasil. $\bowtie$ (iD)
RESUMEN En Brasil, el cuidado en salud mental en los centros de atención primaria se tornó estratégico, dado que garantiza que el usuario asista en su territorio, y la empatía es de gran importancia en el desarrollo de la relación médico-paciente. El objetivo de este estudio fue identificar características relacionadas con la empatía en médicas y médicos que trabajan en el primer nivel y atienden a personas con sufrimiento mental. Se realizó una investigación cualitativa, en 2016, con entrevistas semiestructuradas a ocho médicas y médicos generalistas y de familia. Los relatos se analizaron con la técnica de análisis de contenido y se identificaron tres categorías: empatía en la escucha activa del médico, estrategias que permitan una mejor atención del paciente y movilización de sentimientos. El uso de la empatía está intrínsecamente relacionado con la resolutividad de la atención y las características encontradas fueron la utilización de la escucha activa, el manejo de recursos y el desarrollo de métodos para superar trabas estructurales del cotidiano.

PALABRAS CLAVES Salud Mental; Estrés Psicológico; Atención Primaria de Salud; Empatía; Brasil.

ABSTRACT In Brazil, the provision of mental health care in primary care centers has gained strategic importance, as it ensures that users will be able to attend these services within their communities, and empathy is a key aspect in the development of the doctor-patient relationship. The aim of this study was to identify characteristics related to empathy in physicians who work in primary care centers and who provide care to individuals with mental disorders. Qualitative research was carried out in 2016, based on semi-structured interviews with eight general and family physicians. Material from the interviews was analyzed using content analysis techniques and three categories emerged: empathy in the active listening of the physician, strategies that allow for better patient care, and the mobilization of emotions. The use of empathy is intrinsically linked to the resolutive capacity of care, and the characteristics identified were active listening, resource management, and the development of methods to overcome the structural obstacles of daily routines.

KEY WORDS Mental Health; Psychological Stress; Primary Health Care; Empathy; Brazil. 


\section{INTRODUCCIÓN}

El Sistema Único de Salud (SUS) es el sistema público de atención a la salud en Brasil, de carácter universal, que se sustenta en la atención primaria como uno de los ejes transversales de las redes de atención a la salud ${ }^{(1)}$. Frecuentemente, estas redes se constituyen en el primer contacto del usuario con el servicio y, en ese contexto, la atención primaria en salud mental resulta estratégica, dado que garantiza que el usuario reciba asistencia en su territorio, y facilita que los equipos acompañen a los usuarios y viceversa ${ }^{(2)}$.

Como fuente de seguimiento y con un rol orientador de las acciones de atención primaria, el Ministerio de Salud publica los Cadernos de Atenção Básica, y cada volumen se dedica a una temática relacionada con este nivel de atención. Nuestra trayectoria formativa como estudiantes de medicina y la inserción posterior en vivencias del SUS nos llevó a profundizar y reflexionar respecto del siguiente fragmento publicado en el $\mathrm{Ca}$ derno 34:

Eric Cassell, un importante médico de familia estadounidense, dice que "la evaluación de un sistema de medicina debería ser su adecuación para enfrentar el sufrimiento", y afirma que la medicina moderna falla en este test. $\mathrm{Si}$, por un lado, lidiamos extraordinariamente bien con el dolor, la dificultad para respirar y otras afecciones del cuerpo, fallamos enormemente en lidiar con el sufrimiento de las personas. "Los cuerpos no sufren, las personas sufren", dice Cassell. Gran parte del alivio del sufrimiento que producimos en nuestro trabajo diario deviene de nuestras múltiples facetas como personas, que se visualizan en la interacción con el otro. ${ }^{(2)}$

Vemos que el alcance de la atención primaria es bastante amplio, lo cual exige múltiples habilidades de las y los profesionales de la salud, entre las que cabe destacar la relevancia de la empatía dentro de la producción del cuidado: configura una entidad integrada en el know-how médico, y su demostración sirve para mejorar todo el proceso de cuidado y la propia vida del paciente. La Asociación de Facultades Médicas Americanas declara que los médicos deben ser compasivos y empáticos en el cuidado de los pacientes ${ }^{(3)}$. De forma similar, el segundo principio fundamental del Código de Ética Médica Brasileño explicita que: "II - El objetivo de toda la atención del médico es la salud del ser humano, en beneficio de la cual deberá actuar con el máximo de celo y lo mejor de su capacidad profesional(4)" $^{(4)}$

El campo de la medicina no se dedica exclusivamente a producir y sustentar a los médicos con más conocimientos y habilidades, sino también a los más cuidadosos y empáticos. En ese sentido, la empatía puede definirse como una habilidad que implica entender las experiencias y perspectivas interiores del paciente y una capacidad de comunicar ese entendimiento y actuar a partir de él. Desde la perspectiva de la empatía médica podemos verificar cuatro componentes elementales: el emotivo, el moral, el cognitivo y el comportamental ${ }^{(5)}$.

La empatía afectiva (emocional) explica cómo las personas se tornan conscientes de los estados mentales de otras personas, un estado en el cual el observador toma para sí la emoción del otro, experimentando subjetivamente y compartiendo el estado emocional o los sentimientos de la otra persona, generando una preocupación que precede y contribuye al comportamiento de amparo ${ }^{(6)}$.

La empatía cognitiva es la habilidad de identificar y comprender, en términos objetivos, los sentimientos y las perspectivas de otras personas; es la capacidad de comprender las experiencias de otros sin evocar una respuesta emocional de la persona, por lo que es una competencia que es adquirida y pasible de ser perfeccionada ${ }^{(6)}$.

La empatía comportamental se refiere a la habilidad de transmitirle al paciente que su perspectiva fue comprendida, junto a la habilidad de actuar a partir de ese entendimiento de forma productiva y sin perder la frontera entre el receptor y el emisor, o sea, sin perder 
la consciencia de la diferencia entre sí y el otro. La empatía moral es una motivación interna de preocupación por el otro y un deseo de actuar para aliviar su sufrimiento a través de cuidados y actitudes altruístas ${ }^{(6)}$.

En términos perceptivos, la empatía es un concepto complejo; de modo general y poco específico, se define como una habilidad que permite colocarse en el lugar del otro, de modo de entender su situación actual y, a partir de allí, propiciar una mejor ayuda ${ }^{(7)}$.

En el campo de la salud mental, la empatía permite comprender mejor la experiencia del usuario y favorece la relación médicopaciente, por lo que una falla puede debilitar el vínculo con el usuario. La inmersión empática en el universo psicológico del paciente es una forma de determinar y analizar lo que él realmente experimenta, por lo que el médico debe correrse de su lectura usual del mundo para contextualizar, subjetiva y culturalmente, la experiencia del paciente ${ }^{(8)}$.

Estas actitudes facilitan establecer un vínculo empático en la atención médica, que interfieren al compartir las decisiones, en la adhesión del usuario al tratamiento y en su regreso a las consultas. Además, colaboran en la producción del cuidado en salud mental, valorizando así los recursos comunicacionales como importantes instrumentos para la formación de una buena relación entre los sujetos involucrados en la terapéutica.

El presente estudio forma parte de un proyecto mayor, titulado Sufrimiento mental en la Atención Básica: profesionales, diagnóstico e tratamento en un município de la região Oeste de Santa Catarina, cuyo objetivo fue conocer cómo se abordaba el sufrimiento mental en los servicios de atención primaria del municipio, de la cual ya se ha publicado el artículo "El perfil de usuarios de benzodiazepinas en servicios de atención primaria de la salud de la ciudad de Chapecó, Santa Catarina, Brasil"(11).

Considerando la relevancia de la discusión acerca de la empatía en la producción del cuidado, se buscó identificar características relacionadas con la empatía en médicas y médicos del primer nivel, que atienden a personas con sufrimiento mental.

\section{METODOLOGÍA}

Se realizó un estudio exploratorio, cualitativo, en el que se seleccionaron intencionalmente, como sujetos de investigación, ocho médicas y médicos de cuatro unidades básicas de salud.

Chapecó es un municipio brasileño situado en la región oeste del estado de Santa Catarina y está caracterizado como una ciudad media. En el último censo de 2010 figuraban 183.530 habitantes, de los cuales 90.626 eran hombres y 92.904 mujeres. Al momento de la investigación, la ciudad contaba con 27 centros de salud familiar (CSF) y con 46 equipos de estrategia de salud familiar, distribuidos en las zonas rurales y urbanas de la ciudad, y la cobertura de atención primaria del SUS Ilegaba al 77,12\% de la población ${ }^{(9)}$.

Para determinar los lugares de investigación, inicialmente fue necesario conocer las prescripciones de fármacos psicotrópicos por parte de los médicos generalistas y médicos de familia que trabajaban en el primer nivel de atención del municipio. Para ello, se realizado un relevamiento de los siete principales psicotrópicos cubiertos por el SUS en las seis farmacias del municipio, durante el período de 1 de marzo de 2016 al 31 de mayo 2016. Se seleccionaron las dos unidades de salud con mayor proporción de prescripciones y las dos unidades con menor proporción.

A partir de esa muestra, se realizaron entrevistas individuales semiestructuradas, para lo cual se redactó una guía, en la cual se recabó información de las y los entrevistados (unidad de salud, edad, tiempo transcurrido desde su graduación, tipo de especialización y tiempo de servicio), y se realizaron las siguientes preguntas:

1. Con relación al sufrimiento mental, ipuede describirnos cómo conduce sus consultas, o sea, qué procedimientos adopta?

2. ¿Qué aspectos del paciente son importantes para el diagnóstico de depresión? ¿Qué factores se tienen en cuenta para considerar el diagnóstico? 
3. ¿Qué se puede observar en el paciente? ¿Qué aspectos biológicos, emocionales y comportamentales se consideran?

4. ¿Se solicitan exámenes clínicos? ¿De ser así, cuáles?

5. ¿Cuánto tiempo dura la consulta, en promedio?

6. ¿Como se siente, considerando su formación de médico/a generalista, en el marco de la estrategia de salud familiar, diagnosticando depresión?

7. ¿Desearía conocer los resultados de nuestra investigación? De ser así, ¿cómo desearía recibirlos?

Se realizaron ocho entrevistas a médicas y médicos generalistas y de familia de las unidades seleccionadas en el período de recolección de datos, con un promedio de 30 minutos cada una. Las entrevistas se audiograbaron, se transcribieron y posteriormente se analizaron con base en la técnica de análisis de contenido propuesta por Bardin ${ }^{(10)}$.

El estudio siguió todas las recomendaciones éticas, y fue aprobado por el Comité de Ética en Investigación en Seres Humanos de la Universidade Federal da Fronteira Sul bajo el parecer No. 1.647.056, de 2016 (CAAE: 56468816.5.0000.5564). Todas las personas involucradas en la investigación firmaron los términos del consentimiento libre e informado.

\section{RESULTADOS Y DISCUSIÓN}

A través de la relectura y transcripción de las respuestas de médicas y médicos a las preguntas de la guía de entrevistas, se delimitaron tres categorías: 1) empatía identificada en la escucha activa por parte del médico, 2) estrategias que permitan una mejor atención del paciente y 3 ) movilización de sentimientos. Tales categorías presentan, en sí, características positivas y negativas relacionadas con la empatía, que contribuyen al análisis de esa entidad en el abordaje del sufrimiento mental en atención primaria.

\section{Empatía identificada en la escucha activa por parte del médico}

La empatía identificada en la escucha activa por parte del médico, se configura en demostraciones, incorporadas en los relatos médicos, de una estructuración de la atención basada en la escucha atenta de los anhelos del paciente, que permite una mayor apertura y libertad para la transferencia del propio sufrimiento. Se crea una relación médico-paciente de calidad, al mismo tiempo que se aprovecha una metodología de elaboración de cuidado que permite la afluencia de una gran cantidad de información, dada la confianza provocada en el paciente y la libertad de expresión ofrecida para la demonstración de sus angustias, utilizando el potencial terapéutico que esa interacción puede ofrecer.

\begin{abstract}
Es importante siempre abordar si, por ejemplo, él llora fácilmente, si se siente aislado, solo, sin nadie para conversar; otra cosa importante también es si no tiene voluntad para hacer las cosas, para hacer las actividades cotidianas, trabajar, levantarse a la mañana, si tiene insomnio, si duerme bien a la noche, se ya tuvo ideación suicida, tristeza, ansiedad, también, como está la relación con las otras personas, familiares, en casa; y si eso está afectando su calidad de vida. Si eso afecta su trabajo, las actividades diarias. (Médico 1 - Unidad 4)
\end{abstract}

Algunas acciones que los profesionales del primer nivel de atención pueden realizar son: propiciar instantes para el pensamiento/ reflexión del usuario, practicar una buena comunicabilidad, cultivar la habilidad de la empatía, escuchar aquello que el usuario necesita expresar, recibir sus demandas emocionales como legítimas; promover el apoyo en la medida adecuada, sin tornarlo dependiente y sin ser una carga para el profesional; y distinguir los patrones de comprensión del usuario ${ }^{(2)}$.

De esa forma, se percibe la importancia de ejercer trazos empáticos en la atención de casos relacionados con salud mental. A 
su vez, las actitudes responsables para atender mejor a aquel que sufre evidencian una comprensión acerca de las necesidades del usuario; por lo cual, considerar esas particularidades, considerando la individualidad inherente a la atención de cada persona, exige empatía por parte de aquel que actúa en la producción del cuidado.

En primer lugar, dejo hablar al paciente. Estimulamos al paciente a hablar de todo lo que está sintiendo, los síntomas que está presentando. Después, cuando termina, complementamos con algunas preguntas que, en caso de que falte $o$ en el caso de que no haya hablado, hacemos preguntas para intentar complementar la anamnesis para llegar a un diagnóstico. (Médico 1 - Unidad 3)

¿Cómo que yo converso con el paciente? Él conversa conmigo en realidad, yo soy una buena escuchante. (Médica 1 - Unidad 1)

Consigues componer perfectamente el cuadro de un depresivo, es perfecto así, solo tienes que darle tiempo para hablar.

(Médico 1 - Unidad 1)

El abordaje psicosocial involucra al sujeto en las diversas articulaciones entre lo psíquico y la sociedad, comprende qué relaciones en red marcan nuestra historia de vida, con manifestaciones diarias de las estructuras familiar, social y experiencias culturales, y muestra qué fenómenos biopsicosociales son interdependientes e interactúan entre sí, enmarcados por relaciones entre el sujeto y la red social. Respecto a los trastornos mentales, es necesario superar la concepción de que son meramente de orden individual, desagregados de los demás vivencias del individuo; con acciones que sustentan en responsabilidades compartidas ${ }^{(12)}$.

En los fragmentos presentados se percibe que la anamnesis investiga procesos que superan la queja principal del paciente, y evidencian la existencia de empatía en la relación, dado que tales actitudes muestran preocupación y cuidado. Comprender al individuo más allá de su categoría individual resulta necesario para percibir la realidad del otro, poniendo al médico en una posición de mayor conexión con la persona a la cual presta cuidado.
Así, generalmente se comienza con una conversación para definir bien lo que está sucediendo, si eso está interfiriendo mucho en sus vidas cotidianas, si ya se pensó, por ejemplo, en suicidio o no. A partir de allí, se realiza un amplio abor- daje, de cómo es con la familia, cómo es en el trabajo, en la escuela, además no solo aquello que está incomodando al paciente, sino un abordaje más amplio. Generalmente, si el paciente ya tiene algún sufrimiento, le doy algún tipo de antidepresivo, ya en la primera consulta y, si es necesario, también realizo la deriva- ción a la psicóloga. (Médico 1 - Unidad 4)
¡No, no! ¡No es cerrar un diagnóstico! Así vemos el contexto. (Médico 1 - Uni- dad 4)

Una de las estrategias propuestas en 2003 por la Política Nacional de Humanización del Ministerio de Salud del Brasil fue la escucha calificada, que implica una escucha ampliada, en ambientes apropiados, adaptada a las individualidades de cada usuario. La escucha ampliada o calificada es capaz de estimar las razones que fomentaron acercarse al servicio, reconociendo las necesidades y orientando a la resolución de sus dilemas, a través del manejo de recursos del trabajo en salud, tornando el servicio apto para dar contención y encontrar respuestas más adecuadas para quienes los utilizan ${ }^{(13)}$.

En realidad, lo evaluamos como un todo. No solo, "Ah vino por sufrimiento mental", sino que intentamos abordar otras cosas también, si tiene alguna enfermedad, hipertensión, diabetes, otros tipos de enfermedades, si puede hacerse, si tiene alguna otra enfermedad, si es necesario, pedimos exámenes, a veces, por 
ejemplo, el paciente relata un dolor en el pecho, para excluir alguna causa cardíaca, primero pedimos los exámenes, pero a veces es más evidente que es por una enfermedad mental, ansiedad, pero siempre intentamos excluir otras causas. (Médico 1 - Unidad 4)

Primero, los criterios clínicos. Estos van a valer para cualquier tipo de paciente. Independientemente de la franja etaria, de su escolaridad, de sus ingresos, los criterios clínicos tienen que ser considerados siempre. Pero están también los criterios individuales de cada uno, que es justamente el lugar en el que vive, el trabajo, su cotidiano. Por ejemplo, para diagnosticar una persona con ansiedad, hay que ver si la persona tiene un trastorno de ansiedad generalizada, o si de repente está en la facultad en un período de exámenes, si está con inestabilidad laboral o en un momento que puede ser despedida. Entonces, están los criterios individuales, de cada uno, también: el momento que está atravesando, la familia, el trabajo. (Médico 1 - Unidad 3)

De esa forma, comprender en primer lugar la demanda de la persona que sufre antes de derivarla a otro profesional, brindarle tranquilidad para hablar, Ilorar y abrirse libremente en el momento de la consulta para identificar la real motivación de la demanda de atención y todos los aspectos que subyacen a su relato, son herramientas que establecen una relación médico-persona más empática y constituyen un abordaje más resolutivo.

Respecto del sufrimiento, cuando vemos que la persona sufre, y no siente bienestar, podemos decírselo, entonces, cuando percibimos que tiene una tristeza un poco mayor, que hay angustia, que la ansiedad te imposibilita avanzar, comenzamos a conversar con la persona, ¿verdad? (Médico 1 - Unidad 1)

Afecta el diagnóstico. Si tiene los criterios clínicos bien claros, ya tiene, teóricamente, el diagnóstico. Pero, si tiene algunos criterios clínicos que cierran y otros no, y en la parte personal tiene otras cosas por detrás que lo perjudican, depende. Puede ser que inicie el tratamiento, o no. (Médico 1 - Unidad 3)

Centrar la atención en el relato del paciente y darle el tiempo para que narre su demanda facilita la conexión empática entre el médico y la persona, al mismo tiempo que la función terapéutica permea la escucha calificada.

A veces, es bueno conversar un poquito, intentar entender un poquito más. Si la persona puede hablar ante alguien que la escucha, a veces, mejora también. Es un método terapéutico, lo veo de esa forma. A algunos colegas les gusta hacer el famoso "limpia banco". Atienden super rápido. (Médico 2 - Unidad 3)

El médico de familia y de la comunidad, al actuar en el primer nivel de atención, debería seguir cuatro principios básicos:

Principio I: El médico de familia y de la comunidad es un clínico calificado; principio II: La actuación del médico de familia y de la comunidad está influenciada por la comunidad; principio III: el médico de familia y de la comunidad es un recurso de una población definida; principio IV: la relación médico-persona es fundamental para el desempeño del médico de familia y de la comunidad. ${ }^{(14)}$

A partir de los principios citados, se percibe que el médico de atención primaria debe adaptarse tanto al territorio como a los recursos disponibles en el ambiente donde se inserta, manteniendo una buena relación médico-paciente ${ }^{(2,14)}$. No obstante, las actitudes centradas en mejorar el flujo del sistema también muestran la personalidad empática del médico, extrapolando las dimensiones del paciente atendido de forma individual y abarcando a la comunidad adscrita como un todo. Buscar el carácter resolutivo e integral del cuidado es respetar y saber orientar a los 
usuarios a los diferentes puntos de las redes de atención a la salud, según las necesidades de cada uno ${ }^{(2)}$.

Intentamos resolver la mayor cantidad de cosas aquí dentro de la unidad. Cuando no lo conseguimos nosotros, los casos más graves, depresión grave, esquizofrénicos, bipolares que están con brotes psicóticos, debemos derivarlos al CAPS I/ o para internación en el hospital para salir de la crisis como en los casos de suicidio, de que mate a alguien, en fin, cuando está en un brote y después regresa con nosotros. (Médico 3 - Unidad 3)

Porque si derivamos todo, las personas que realmente necesitan van a tener demora para llegar hasta allá. (Médico 1 Unidad 4)

Contraponiendo los relatos, se puede percibir que cuando falta establecer una conexión empática en la relación médico-persona, por un deseo de resolutividad, el médico identifica indicios de sufrimiento mental y se siente más seguro al derivar al usuario que al buscar diferentes estrategias en el plano terapéutico e instituir una relación más profunda, un diálogo. Otro contrapunto es el hecho de reducir el sufrimiento a una causa pasible de resolución con medicamentos, sin recurrir a herramientas valiosas como una visita domiciliaria, terapias de grupo o prácticas integrativas y complementarias.

Vale destacar que el acuerdo propuesto por la Red de Atención Psicosocial en Brasil cuenta con centros de atención psicosocial (CAPS), que son los puntos especializados de atención para el usuario que presenta sufrimiento mental, y actúan de forma multiprofesional e interdisciplinaria para promover la receptividad dentro de un modelo adaptado a la realidad social brasileña, que surgió como sustitución al modelo asilar, además de proporcionar apoyo matricial a los profesionales de atención primaria( ${ }^{(2)}$.
No, no. Nunca hice una visita domiciliaria. Conozco algunas calles aquí, pero poco. (Médico 1 - Unidad 3)

Además, algunos de los relatos analizados separan la dimensión física del paciente de la mental, lo que muestra una falta de asociación entre las habilidades clínicas objetivas, utilizadas para analizar los aspectos de la salud corporal y las habilidades de comprensión de aspectos psicológicos. En estos casos, el recurso de la empatía no fue adecuadamente empleado para asociar todas las dimensiones de la persona a la cual se presta cuidado. Estos aspectos muestran que la educación médica está permeada por la reforma flexneriana, que priorizó el cientificismo y la hiperespecialización dentro de la formación, por lo que es necesario enfatizar la humanización dentro del currículo médico(15).

A pesar de las divergencias en la bibliografía, la mayoría de los estudios del área señalan una reducción del nivel de empatía en estudiantes de medicina en el transcurso de la carrera, principalmente, en tercer año, al iniciarse la etapa clínica. En este sentido, se describen distintos mecanismos como: la protección ante situaciones arduas y emocionalmente exigentes, inherentes al cuidado de la salud; el fenómeno de adaptación, por medio del cual la idealización inicial de ser médico al inicio de la carrera se sustituye por la noción de que el éxito equivale a la apropiación masiva de contenidos y el buen desempeño en los exámenes, descuidando habilidades de comunicación o empáticas ${ }^{(16,17)}$.

También se describe agotamiento relacionado con la compasión para enfrentar las molestias, las angustias y la muerte, y un alto grado de responsabilidad intrínseca a la actividad pueden desencadenar malestar psíquico y emocional; y el sufrimiento mental vinculado a innumerables aspectos relacionados con la facultad y la propia profesión, que pueden incluso desencadenar cuadros de síndrome de burn-out ${ }^{(16,17)}$. Otro aspecto importante a considerar es el propio currículo académico, dado que la formación tradicional prioriza la dimensión intelectual, tecnicista y cientificista, instrumentaliza el 
proceso comunicacional y la sobrecarga os estudiantes globalmente. A su vez, hay muchos relatos de los estudiantes respecto del trato que reciben de sus colegas y superiores: no muestran la empatía y el respeto adecuados por parte de docentes y equipos hospitalarios; además de la falta de modelos positivos de la praxis profesional, ausencia de apoyo social por parte de los colegas o del núcleo familiar; entre otros ${ }^{(16,17)}$.

Un abordaje adecuado de la empatía dentro de la formación médica es una herramienta de intervención para evitar su disminución y promover su utilización como herramienta terapéutica. Con ese objetivo, las directrices curriculares nacionales que orientan las carreras de medicina promovieron reestructuraciones curriculares, entre las cuales está previsto el abordaje de habilidades empáticas y comunicacionales a lo largo de la carrera ${ }^{(16,17,18)}$.

\section{Estrategias que permiten atender mejor al paciente}

Entre las estrategias que permiten una mejor atención del usuario, se incluyen discusiones acerca de las tácticas utilizadas en el cotidiano médico que permitan mitigar desventajas encontradas por limitaciones estructurales del ambiente de trabajo; eso aumenta el vínculo entre el médico y el paciente, mejora la extensión del seguimiento, y proporciona al atendido una experiencia que corresponda a sus necesidades y particularidades.

En comparación a las otras áreas de la medicina, el campo de la salud mental es único en el sentido de que el cuidado se brinda a pacientes que tienen distintos niveles de consciencia, creencias y comprensión acerca de su enfermedad; una alteración puede afectar su habilidad de confiar, entender o discernir para llegar a una decisión ${ }^{(19)}$. En el cuidado médico, la empatía ayuda al desarrollo de una relación efectiva entre las partes, permite no descuidar ciertas particularidades clínicas de la persona a la cual se presta cuidado, que podrían no ser descifradas nunca de otra forma, o que al ser tan sutiles, podrían pasar desapercebidos. Además, ofrece el soporte necesario para transmitir información difícil de procesar, como la comunicación de malas noticias, y el soporte para su recuperación ${ }^{(20)}$.

En esa conversación, me olvido de la computadora, me olvido de todo, siempre me siento un poco más cerca del paciente, hasta para tener un mejor contacto, cuando el paciente te permite eso ¿no?, siempre eso, y converso con el paciente. (Médico 1 - Unidad 1)

Es una cuestión del vínculo, de la relación, vamos a decir, médico-paciente, del equipo-paciente ¿no? En la relación médico-paciente, el paciente viene aquí y habla un poquito, en la segunda consulta ya habla un poquito más, así lo voy conociendo un poco más, y así se va estableciendo una relación de confianza también, entre el médico y el paciente, para que también se de adhesión al tratamiento. (Médico 1 - Unidad 2)

El uso de estrategias que posibiliten una mejor atención al paciente se torna un mecanismo para desarrollar diversas dimensiones empáticas: la cognitiva, que manifiesta comprensión de la condición en la cual se encuentra quien recibe el cuidado; la moral, que moviliza el interés y el deseo interior de aplicar conductas que apuntan a reducir la aflicción; y la comportamental, que evoca actitudes productivas que demuestran esa comprensión y se adaptan a las particularidades del contexto. El recurso de colocarse más cerca de la persona, eliminando barreras físicas (como computadoras y mesas), promueve la receptividad y la comodidad y facilita la conexión empática. Actitudes como esta promueven un ambiente acogedor, aspecto recomendado dentro de la atención primaria por la Política Nacional de Humanización ${ }^{(21)}$. Los relatos que proponen dedicar más tiempo a las consultas, y dejar que el usuario se exprese a voluntad, también corroboran esa idea. 
Yo aumento el tiempo, ahí sé que voy a poder prestar más atención. ¡No va a ser algo ajustado! Ella viene y vamos a conversar, vamos a hablar, entonces le damos un poquito más de tiempo, y eso ayuda bastante. (Médico 1 - Unidad 1)

Las estrategias discutidas en los fragmentos posibilitan una mayor cercanía al paciente, estableciendo una mayor conexión y un diálogo más empático. Tales factores son relevantes para un mejor entendimiento de la persona por parte del médico y, por consiguiente, para una mejor conducta frente al caso. La reevaluación constante también es una forma de comprender mejor la situación del paciente, y puede ser considerada un indicio de la presencia de empatía dentro de la consulta. Considerando la importancia de la empatía dentro de la relación médico-paciente, se torna imperativo adoptar actitudes que mejoren el desarrollo del vínculo empático y, en consecuencia, hagan que esa interacción sea más satisfactoria y asertiva.

Esto es de suma relevancia dentro de un contexto que moviliza diversos sentimientos en el paciente, por el propio carácter de la consulta por sufrimiento mental. De esa forma, que tanto el equipo como el propio ambiente demuestren receptividad, privacidad y consuelo son factores elementales del abordaje de este tipo de consultas. La comprensión de estos aspectos muestra los elementos empáticos que se desencadenan en los médicos, quienes manifiestan ese entendimiento como regla en la atención de esos casos.

Eso, siempre se hace una reevaluación. (Médico 1 - Unidad 4)

...si tiene riesgo de suicidio [...] independientemente de si el tratamiento que inicié va a resultar o no, ya se hace la derivación al psiquiatra. (Médico 1 - Unidad 3)

...le pido al paciente que regrese en un mes [...] si tuvo algún efecto adverso, siempre le indico que regrese antes [...] si no dio, o si tuvo algún efecto colateral, cambio la fecha. Pero siempre les digo que regresen dentro de un mes o dos.

(Médico 1 - Unidad 4)

Las necesidades de salud se manifiestan en diversas dimensiones; por lo tanto, las demandas no pueden ser sanadas a partir de la acción aislada de un profesional ${ }^{(22)}$. De esa forma, es imprescindible destacar el trabajo del equipo multiprofesional, dado que una estrategia relatada es precisamente pedir a otro profesional de la unidad que ampare al paciente en el caso de que la consulta se prolongue mucho. Esa práctica resulta válida dado que aumenta la receptividad del paciente, mejora el vínculo con el médico y el equipo y ayuda a instaurar la empatía ${ }^{(19)}$.

...le pasa a la enfermera "estuve media hora y no puedo hacer más nada", [...] Seguimos hablando con la paciente porque no se la puede dejar salir de aquí así [...]. Y cuando no conseguimos calmarla, Ilamamos a un familiar también. (Médico 1 - Unidad 1)

Las estrategias tanto para lidiar con el tiempo limitado de la consulta y con la falta de psicólogos, como para conocer los medicamentos disponibles en la unidad y recurrir a herramientas como la visita domiciliaria y grupos terapéuticos, como los de desarrollo humano, son formas de tornarse resolutivo $y$, principalmente, empático, dado que demuestran esfuerzo por parte del médico para ayudar a aquel que lo procura a pesar de las limitaciones impuestas por el sistema.

...básicamente es eso, se intenta con todos los medicamentos que hay aquí en la farmacia, en la unidad y si no resulta, psiquiatra. (Médico 1 - Unidad 3)

En promedio, 10 a 15 minutos. No hay cómo prolongar más porque estoy atendiendo al paciente y hay ocho ahí afuera esperando. Entonces, no se puede. Si necesito más tiempo, dependiendo del caso, agendamos una nueva fecha, o entonces, para casos específicos intento darles un poquito más de tiempo, pero 
no hay cómo extenderse de 15 minutos.

Si no el paciente agendado para las 9 sería

atendido a las 11. (Médico 1 - Unidad 3)

Resulta más visible el contrapunto cuando no hay estrategias por parte del médico para optimizar la atención y alcanzar una mejor aproximación al paciente. A pesar de no ser una actitud incorrecta, configura un indicio de escasa empatía por parte del profesional, dado que incluso ante situaciones más complejas, no se concede más tiempo o no se incorporan otras estrategias, como la ayuda del equipo.

\section{Movilización de sentimientos}

En esta categoría se presentan relatos en los que se encuentran demostraciones controladas de afecto que involucran al paciente, su sufrimiento y la oferta de cuidado ligada a una relación empática entre médico y persona. De ese modo, configuran un punto importante en el abordaje y la comprensión del sufrimiento del prójimo, que se torna una herramienta fundamental en la terapéutica. Esa categoría presenta la demonstración de la dimensión emocional de la empatía, en la que el receptor de las informaciones experimenta subjetivamente la condición sentimental y emocional de aquel que emite su perspectiva.

Sí, los primeros muertos no se olvidan. No sos el responsable, pero estabas ahí presente, intentaste salvarlo y no lo conseguiste. Eso, al inicio, es obvio, tenemos que pasar por varias experiencias para que te acostumbres, no te acostumbras, pero no dejas que afecte tu vida. Porque primero está tu profesión. Yo elegí esta profesión, necesito acostumbrarme a las tragedias. En realidad, diariamente, trabajamos solo con enfermedad. (Médica 3 - Unidad 3)

La relación médico-paciente es un acuerdo asimétrico de roles complementarios, donde el primero debe cumplir tareas instrumentales relacionadas con el examen y tratamiento; mientras el segundo tiene preocupaciones relacionadas con la salud. A pesar de todo, ese grado de asimetría puede modificarse en ciertas consultas, especialmente cuando el usuario manifiesta dilemas emocionales. La simetría dentro de la comunicación aumenta, junto al grado de mutualidad. Estos encuentros se describen frecuentemente como momentos de "conexión", intensos en compasión y compañerismo, que suelen estar acompañados de reacciones fisiológicas como lágrimas en los ojos o escalofríos. Los roles de cada uno, teóricamente, se conservan intactos; pero se altera el patrón del diálogo ${ }^{(23)}$.

Entre las cuatro dimensiones de la empatía, en este caso, se destaca la empatía afectiva. La batalla para equilibrar la conexión emocional -la empatía afectiva- y la preocupación profesional imparcial -empatía cognitiva- en la relación médico-paciente es una constante entre los médicos. Una relación empática con la persona que es atendida fomenta la confianza y le permite confesar sus más profundos miedos. La empatía implica sentir con el paciente para alcanzar una comprensión de su sufrimiento y que el médico exponga inevitablemente su vulnerabilidad, lo cual requiere dividir una parte de sí con la otra persona. La preocupación empática es compartir emociones: el médico siente el dolor del paciente mientras es consciente del límite entre sí y el otro; que es posible diferenciar de la angustia personal, que resulta de tomar una perspectiva autoorientada (“¿cómo me sentiría en esa situación?"), que puede llevar a una identificación y consiguiente sobrecarga ${ }^{(24)}$.

Los médicos pueden sentir temor ante una conexión emocional por la preocupación de que su juicio clínico sea menos objetivo o que queden sobrecargados y agotados por la situación, lo que puede producir un distanciamiento del paciente, como si el desapego formara parte de ser profesional. Un aspecto primordial de esa dimensión de la empatía es compartir sentimientos, en vez de meramente rotular un estado emocional. El distanciamiento no es necesario para un juicio clínico confiable, dado que las intuiciones 
emocionales pueden orientar la toma de decisiones. A su vez, los médicos empáticos tienen una mayor satisfacción profesional y menor agotamiento; incluso si son capaces de suprimir sus sentimientos, no pueden evitar tomar actitudes emocionales frente a los pacientes $^{(24)}$.

Tengo, como médica, tengo una visión muy clara de la medicina. Tengo mi parte [...] de ser humano, pero consigo posicionarme profesionalmente $y$ demostrar el soporte y el apoyo que el paciente está necesitando de mí. Si me deprimo con él, el paciente no se va a tratar conmigo. Entonces, yo complemento como médica. Tengo la visión de la enfermedad de una forma muy tranquila, soy médica, necesito ser así, para poder curar, ayudar a las personas a tratarse. Entonces, no llevo los problemas de aquí a mi vida personal. Me pongo triste, a veces, cuando muere un paciente en casos graves, así, el paciente se suicidó, tengo sentimientos humanos como ellos, con los familiares, pero no los Ilevo conmigo. No los llevo para mí. Si no, me mato cuando llego a casa.

(Médica 3 - Unidad 3)

Los relatos ejemplifican las formas de expresión de afecto por parte del médico para con sus pacientes $y$, por consiguiente, la demonstración subjetiva de empatía. A partir del análisis, se pudo percibir que aquellos médicos que manifestaron más actitudes empáticas dentro de su rutina, fueron los que movilizaron más sentimientos.

Amo a mis pacientes y les tengo un cariño enorme. Levo para la parte de la humanización de la medicina. (Médico 3 - Unidad 3)

Estamos en la salud para compartir, no estamos en salud para ganar dinero, o algo así, ¿entiendes? Entonces, tienes que saber hacer eso, tienes que saber cuidar al prójimo, ¡eso es necesario! Si no sabes cuidar al prójimo, sos extremamente incompetente, ¡extremamente! (Médico

1 - Unidad 1)

En el modelo de empatía de Irving, el médico necesita entender el mundo del paciente ( $\operatorname{cog}$ nitivo), sentir con él (afectivo) y comunicar esa comprensión (comportamental) ${ }^{(25)}$. La empatía es un proceso dinámico, que excede la "compasión" que suele ser requerida de los médicos: se trata de un conjunto de habilidades, actitudes y preocupación moral. Aceptar -en oposición a resistir sus propias emociones- les permite a los médicos practicar el cuidado sin aflicciones. Involucrarse implica algún grado de identificación de la persona como un ser humano, con las mismas necesidades, y que merece el mismo respeto ${ }^{(6)}$.

De este modo, la movilización de sentimientos que se observa en los fragmentos, la habilidad de preocuparse por quien tengo delante, muestra un compromiso real con el cuidado del otro y una identificación del otro como un igual; eso se traduce en habilidades que permiten una mejor atención de quien procura ayuda. Perceptiblemente, la empatía no es algo "extra" al cuidado, es facultad del profesional de salud tenerla o no. Es absolutamente esencial para todo el proceso de recepción, atención, entrevista, diagnóstico, terapéutica y vínculo; por lo tanto, es esencial que, quien lleva adelante el proceso, la ejerza diaria y constantemente. La empatía no es algo que sucede, es una elección y exige esfuerzo ${ }^{(5)}$.

\section{CONCLUSIÓN}

El desarrollo y la mejora de la comunicación en salud mental requiere un esfuerzo continuo de los profesionales, y la empatía se torna primordial en la divulgación tecnológica de información que atraviesa la medicina, otorgando un papel importante al cuidado en salud. Las actitudes empáticas acarrean más receptividad, vínculo, confianza, comodidad y bienestar $y$, a su vez, permite una postura más resolutiva, principalmente en lo referente a las personas con sufrimiento mental, dado 
que proporciona más comodidad al usuario, que da como resultado un mayor vínculo y participación en el contrato terapéutico.

Los hallazgos de este artículo evidencian que los médicos de atención primaria en Chapecó asocian las siguientes características a la empatía: la utilización de la escucha activa como método para ampliar la perspectiva y asimilar el contexto psicosocial del sujeto, el manejo de los recursos disponibles para la atención y encontrar métodos que permitan superar las trabas estructurales del cotidiano. De este modo, la empatía resulta ser un recurso que modifica la atención, con respaldo científico y compleja aplicabilidad práctica.

\section{REFERENCIAS BIBLIOGRÁFICAS}

1. Melo EA, Mendonça MHM, Oliveira JR, Andrade GCL. Mudanças na Política Nacional de Atenção Básica: entre retrocessos e desafios. Saúde em Debate. 2018;42(Spe 1):38-51. doi: 10.1590/0103-11042018s103.

2. Brasil, Ministério da Saúde. Saúde mental. Cadernos de Atenção Básica [Internet]. Brasília: Ministério da Saúde; 2013 [citado 10 abr 2019]. Disponible en: https:// tinyurl.com/yysu4edu.

3. Association of American Medical Colleges. Report I: Learning objectives for medical student education, Guidelines for Medical Schools [Internet].1998 [citado 10 abr 2019]. Disponible en: https://tinyurl.com/yyx3ktob.

4. Conselho Federal de Medicina. Código de Ética Médica 2009 [Internet]. Brasília: Conselho Federal de Medicina; 2009 [citado 8 may 2019]. Disponible en: https://tinyurl.com/yy2gln3m.

5. Kelm Z, Womer J, Walter JK, Feudtner C. Interventions to cultivate physician empathy: a systematic review. BMC Medical Education. 2014;14:219. doi: 10.1186/14726920-14-219.

6. Jeffrey D. Empathy, sympathy and compassion in healthcare: is there a problem? is there a difference? does it matter? Journal of the Royal Society of Medicine. 2016;109(12):446-452. doi: 10.1177/0141076816680120.

7. Hemmerdinger JM, Stoddart SDR, Lilford RJ. A systematic review of tests of empathy in medicine. BMC Medical Education. 2007;7:24. doi: 10.1186/14726920-7-24.

8. Peixoto $M M$, Mourão $A C N$, Serpa Junior $O D$. Coming to terms with the other's perspective: empathy in the relation between psychiatrists and persons diagnosed with schizophrenia. Ciência \& Saúde Coletiva. 2016;21(3):881-890. doi: 10.1590/141381232015213.04782015 .

9. Instituto Brasileiro de Geografia e Estatística. Cidades: Chapecó [Internet]. 2017 [citado 2 jul 2020]. Disponible en: https://tinyurl.com/y6gjp68w.

10. Bardin L. Análise de conteúdo. São Paulo: Edições 70; 2011.
11. Oliveira-Friestino JK, Luzardo AR, Gralak EZ, Gass LS, Guimarães PGZ, Barbato PR. El perfi I de usuarios de benzodia-zepinas en servicios de atención primaria de la salud de la ciudad de Chapecó, Santa Catarina, Brasil. Salud Colectiva. 2020;16:e2495. doi: 10.18294/ sc. 2020.2495

12. Alves ES, Francisco AL. Ação psicológica em saúde mental: uma abordagem psicossocial. Psicologia: Ciência e Profissão. 2009;29(4):768-779. doi: 10.1590/S141498932009000400009.

13. Duarte LP, Moreira DJ, Duarte EB, Feitosa AN, Oliveira AM. Contribuição da escuta qualificada para a integralidade na atenção primária. Revista Eletrônica Gestão \& Saúde. 2017;8(3):414-419. doi: 10.18673/ gs.v8i3.24185.

14. Gusso G, Lopes JMC. Tratado de medicina de família e comunidade: princípios, formação e prática. Porto Alegre: Artmed; 2012.

15. Neves NMBC, Neves FBCS, Bitencourt AGV. O ensino médico no Brasil: origens e transformações. Gazeta Médica da Bahia. 2005;75(2):162-168.

16. Schweller M, Wanderlei J, Strazzacappa M, Sá FC, Celeri EHRV, Carvalho-Filho MA. Metodologias ativas para o ensino da empatia na graduação em medicina - Uma experiência da UNICAMP. Cadernos ABEM. 2014;10:36-46.

17. Madeira L, Silva HM. Empatia e competências empáticas no curso de medicina: aspetos conceptuais para a sua preservação e promoção. Revista Jurídica LusoBrasileira. 2020;6(1):475-504.

18. Brasil, Ministério da Educação. Resolução CNE/ CES 3/2014: Diretrizes curriculares nacionais do curso de graduação em medicina [Internet]. 23 jun 2014 [citado 30 jul 2020]. Disponible en: https://tinyurl.com/ yxlyr7kc.

19. Harbishettar V, Krishna KR, Srinivasa P, Gowda $M$. The enigma of doctor-patient relationship. Indian Journal of Psychiatry. 2019;61(Suppl 4):S776-S781. doi: 10.4103/psychiatry.IndianJPsychiatry_96_19.

20. Borkar S. Empathy in physician-patient relationship: The construct and Its applicability to India's Health Care. Social Change. 2014;44(3):423-438. 
21. Brasil. Ministério da Saúde, Secretaria de Atenção à Saúde. HumanizaSUS: Política Nacional de Humanização da Saúde [Internet]. Brasília: Ministério da Saúde; 2003 [citado 15 jun 2019]. Disponible en: https://tinyurl. com/y5z7b6va.

22. Schraiber LB, Peduzzi M, Sala A, Nemes MI, Castanhera ER, Kon R. Planejamento, gestão e avaliação em saúde: identificando problemas. Ciência \& Saúde Coletiva. 1999;4(2):221-242.

23. Finset A, Ørnes K. Empathy in the clinician-patient relationship: The role of reciprocal adjustments and processes of synchrony. Journal of Patient Experience. 2017;4(2):64-68. doi: 10.1177/2374373517699271.

24. Jeffrey D. Communicating with a human voice: developing a relational model of empathy. Journal of the Royal College of Physicians of Edinburgh. 2017;47(3):266270. doi: 10.4997/JRCPE.2017.312.

25. Irving P, Dickson D. Empathy: Towards a conceptual framework for health professionals. International Journal of Health Care Quality Assurance Incorporating Leadership in Health Services. 2004;17(4-5):212-220. doi: 10.1108/09526860410541531.

\section{FORMA DE CITAR}

Friestino JKO, Luchini Junior D, Lange Biesek L, Marcon G, Fonsêca GS. Comunicación y salud mental: características relacionadas con la empatía en médicas y médicos del primer nivel de atención en Chapecó, Santa Catarina, Brasil. Salud Colectiva. 2020;16:e3034. doi: 10.18294/sc.2020.3034.

Recibido: 1 ago 2020 | Versión final: 4 nov 2020 | Aprobado: 24 nov 2020 | Publicado en línea: 20 dic 2020

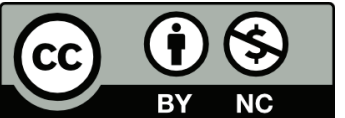

Esta obra está bajo una licencia de Creative Commons Reconocimiento-NoComercial 4.0 Internacional. Reconocimiento - Permite copiar, distribuir y comunicar públicamente la obra. A cambio, se debe reconocer y citar al autor original. No Comercial - Esta obra no puede ser utilizada con finalidades comerciales, a menos que se obtenga el permiso. 